\title{
Improving the Description of the Optical Properties of Carotenoids by Tuning the Long-Range Corrected Functionals
}

Igo T. Lima,${ }^{1,2}$ Andriele da S. Prado, ${ }^{1}$ João B. L. Martins, ${ }^{3}$ Pedro H. de Oliveira Neto, ${ }^{1}$ Artemis M. Ceschin, ${ }^{4}$ Wiliam F. da Cunha ${ }^{1}$ and Demétrio A. da Silva Filho. ${ }^{1, *}$

${ }^{1}$ Institute of Physics, University of Brasilia, 70919-970, Brasilia, Brazil.

${ }^{2}$ Federal University of Maranhão, 65800-000, Balsas,_Maranhão, Brazil.

${ }^{3}$ Institute of Chemistry, University of Brasilia, 70919-970, Brasilia, Brazil.

${ }^{4}$ Electrical Engineering Department, University of Brasilia, 70919-970, Brasilia, Brazil. 


\section{List of Figures \& Tables.}

Figure S1. Tuning of the range separation parameter $(\omega)$ for the ionization potential, electron affinity and gap of the phytofluene.

Figure S2. Illustration of frontier molecular orbitals HOMO (left) and LUMO (right) determined at tuned LC-BLYP/6-31G(d,p) level of theory.

Figure S3. Illustration of frontier molecular orbitals HOMO (left) and LUMO (right) determined at tuned $\omega \mathrm{B} 97 / 6-31 \mathrm{G}(\mathrm{d}, \mathrm{p})$ level of theory.

Figure S4. Absorption spectra for the five molecules computed at tuned $\omega$ B97/6-31G(d,p) level of theory.

Table $\mathrm{S} 1 . \mathrm{S}_{0} \rightarrow \mathrm{S}_{1}$ vertical transition energies $\left(\mathrm{E}_{01}\right)$, oscillator strengths $(f)$, transition dipole moments $\left(\mu_{01}\right)$, and electronic configurations of carotenoids as determined with TDDFT at the tuned- $\omega$ B97/6-31G(d,p) level of theory. 


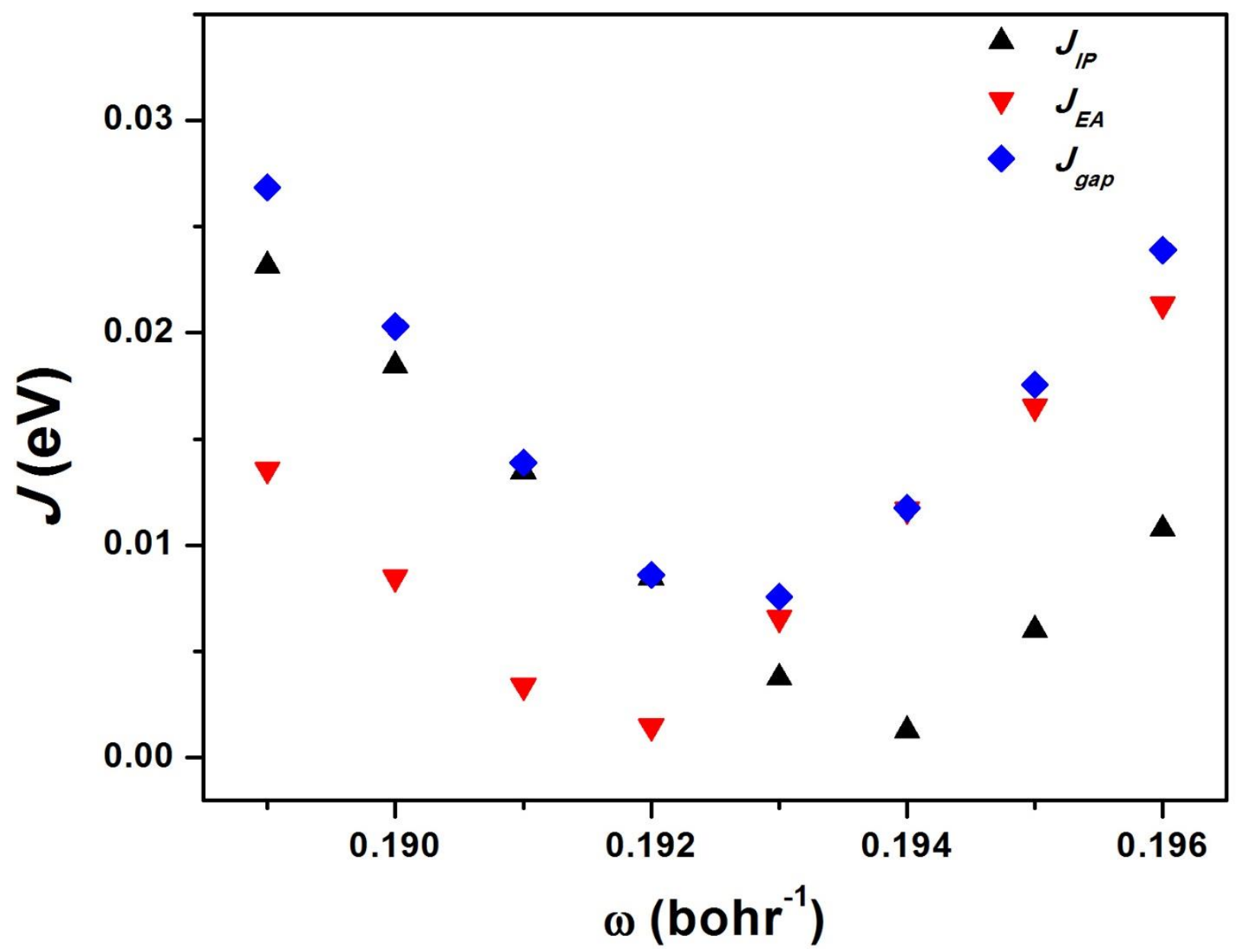

Figure S1. Tuning of the range separation parameter $(\omega)$ for the ionization potential, electron affinity and gap of the phytofluene. 
HOMO

\section{3-cis- $\beta$-carotene}

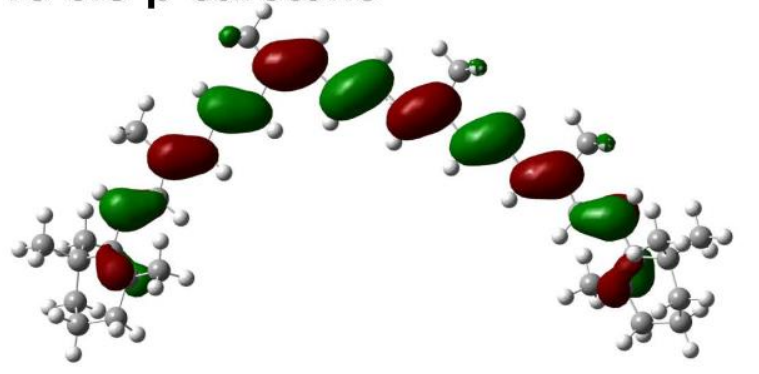

LUMO

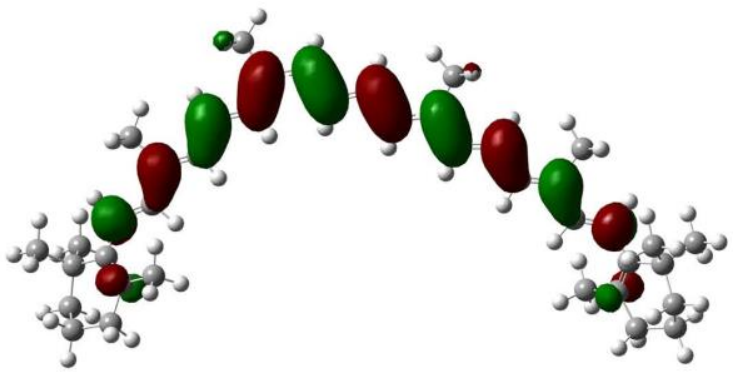

\section{9-cis- $\beta$-carotene}
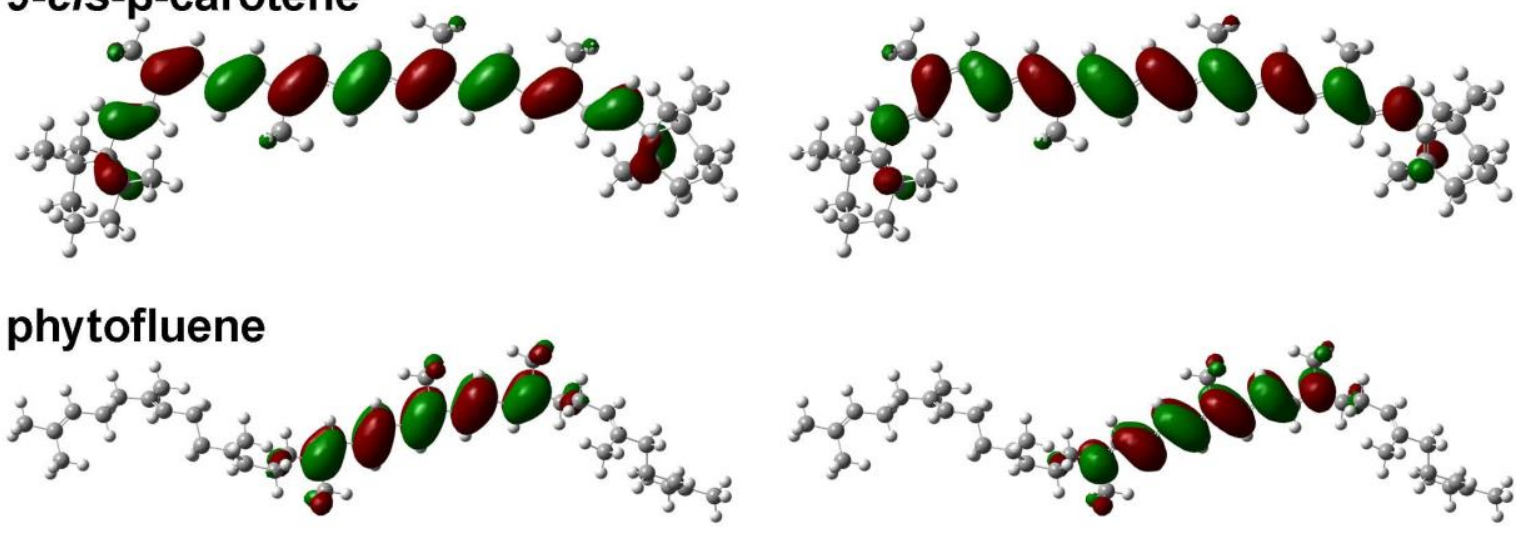

\section{trans- $\beta$-carotene}
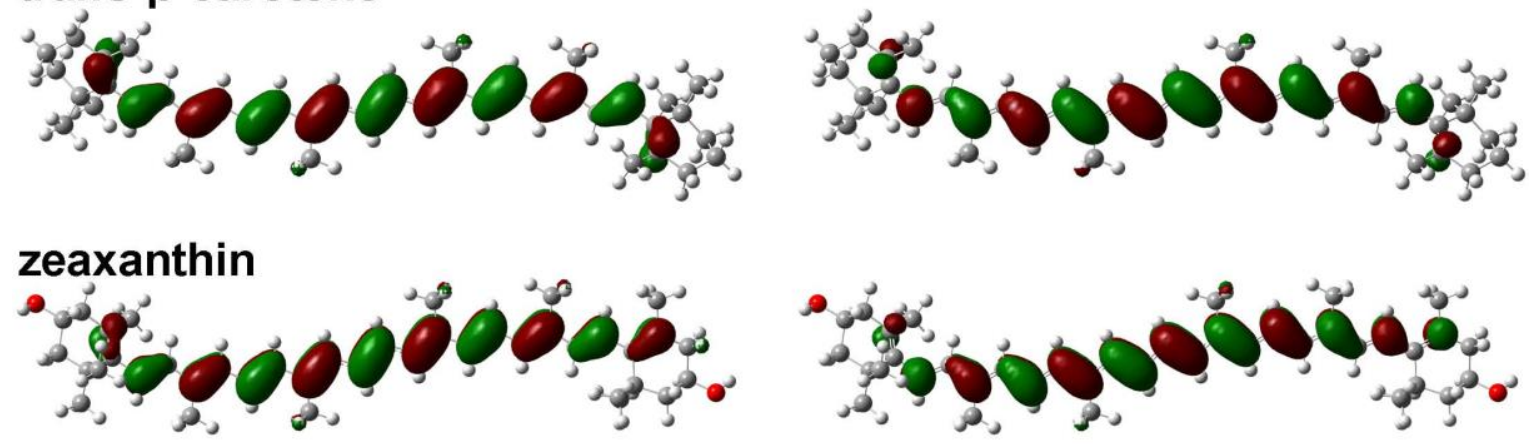

Figure S2. Illustration of frontier molecular orbitals HOMO (left) and LUMO (right) determined at tuned LC-BLYP/6-31G(d,p) level of theory. 
HOMO

13-cis- $\beta$-carotene

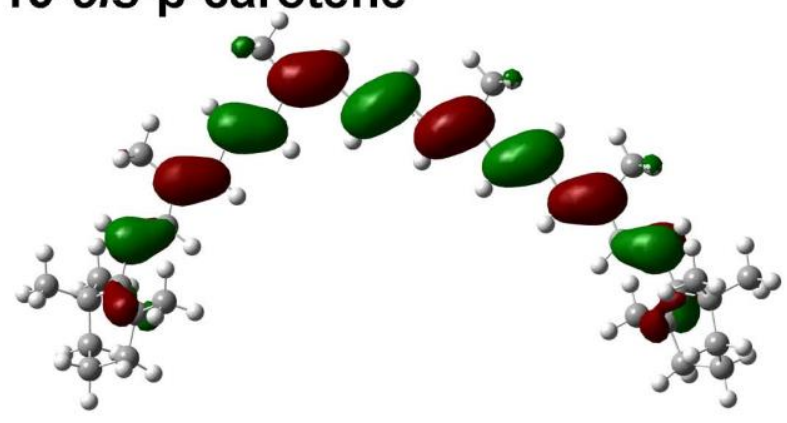

LUMO

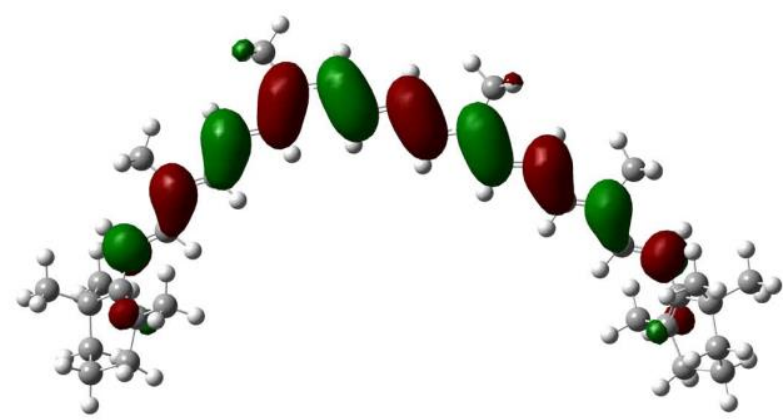

\section{9-cis- $\beta$-carotene}
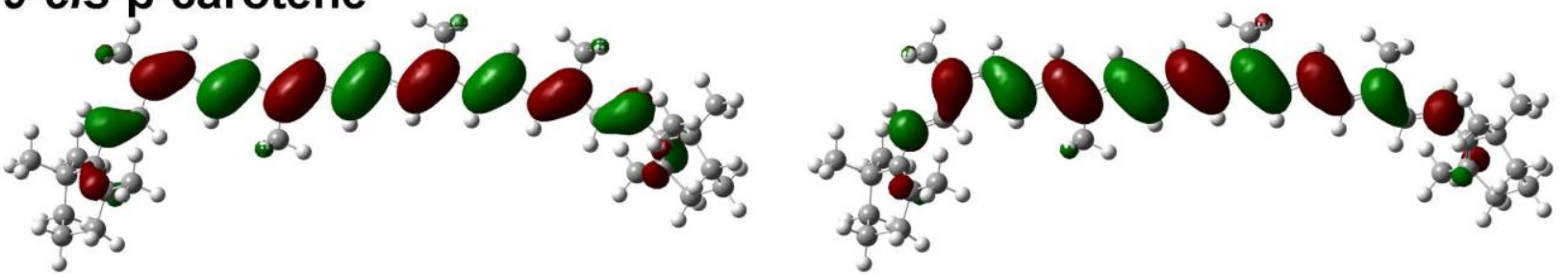

phytofluene

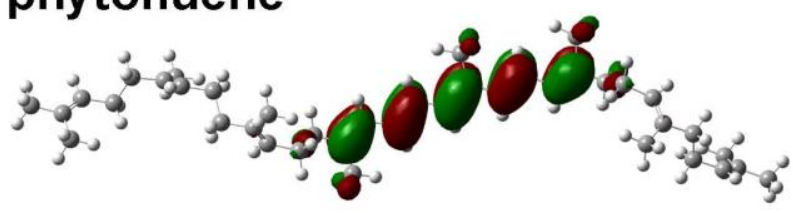

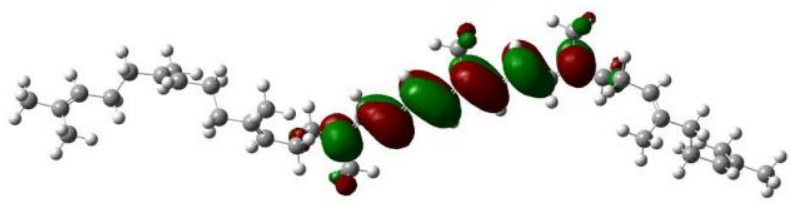

\section{trans- $\beta$-carotene}
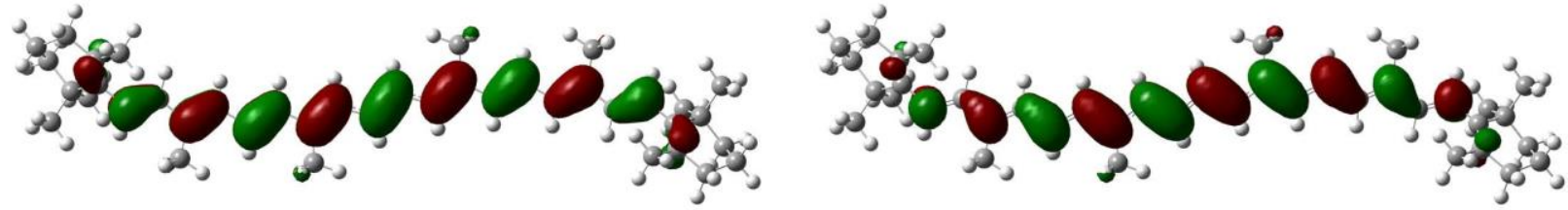

zeaxanthin
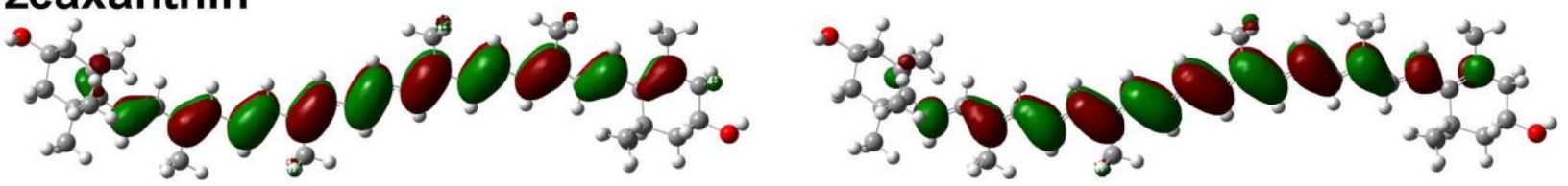

Figure S3. Illustration of frontier molecular orbitals HOMO (left) and LUMO (right) determined at tuned $\omega \mathrm{B} 97 / 6-31 \mathrm{G}(\mathrm{d}, \mathrm{p})$ level of theory. 


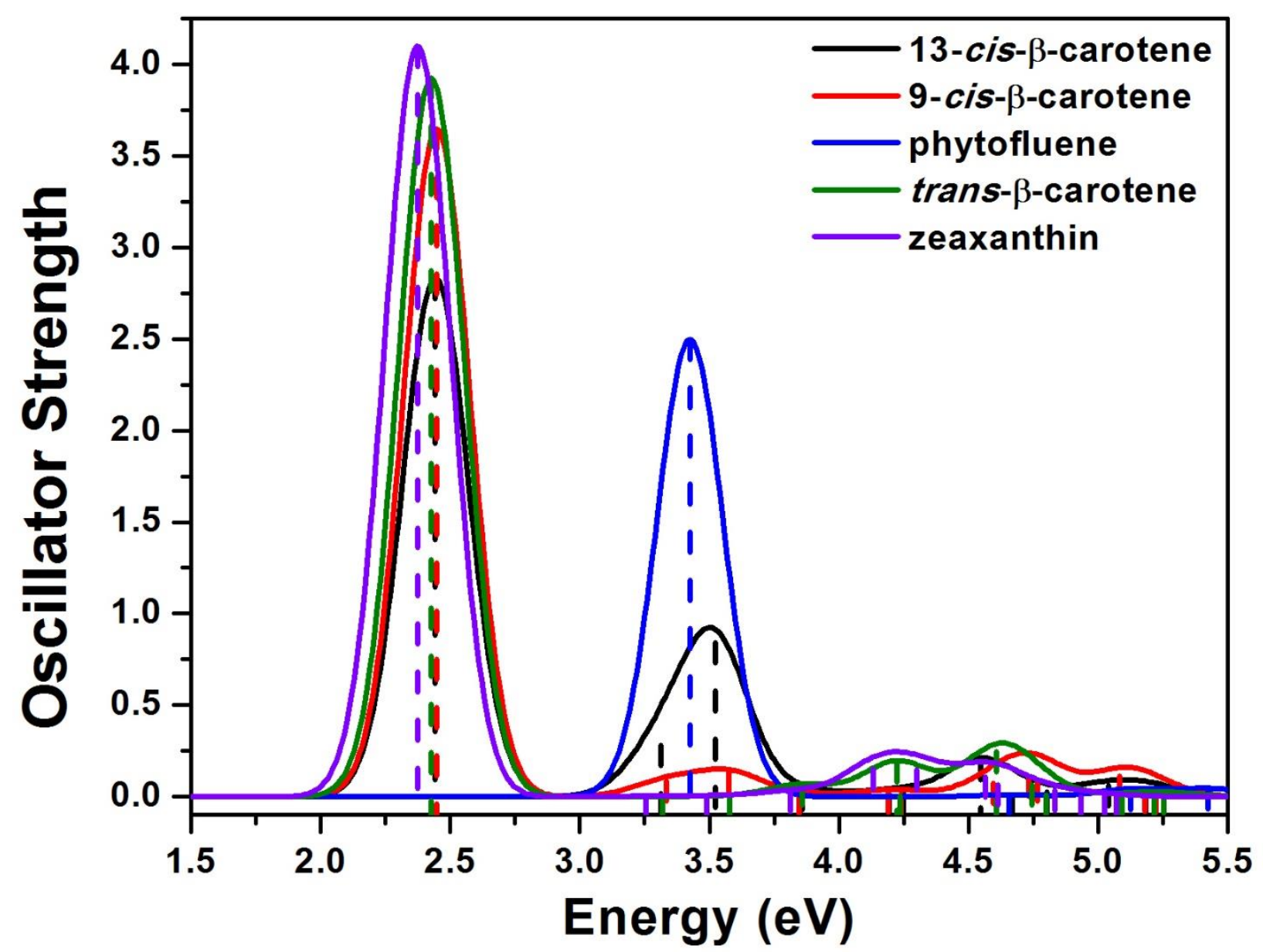

Figure S4. Absorption spectra for the five molecules computed at tuned $\omega \mathrm{B} 97 / 6-31 \mathrm{G}(\mathrm{d}, \mathrm{p})$ level of theory.

Table S1. $\mathrm{S}_{0} \rightarrow \mathrm{S}_{1}$ vertical transition energies $\left(\mathrm{E}_{01}\right)$, oscillator strengths $(f)$, transition dipole moments $\left(\mu_{01}\right)$, and electronic configurations of carotenoids as determined with TDDFT at the tuned- $\omega$ B $97 / 6-31 G(d, p)$ level of theory.

\begin{tabular}{|c|c|c|c|c|c|c|c|}
\hline \multirow{2}{*}{ Molecule } & \multirow{2}{*}{$\begin{array}{c}\mathrm{E}_{01} \\
(\mathrm{eV})\end{array}$} & \multirow{2}{*}{$f$} & \multicolumn{4}{|c|}{$\mu_{01}$ (Debye) } & \multirow{2}{*}{$\begin{array}{c}\text { Electronic } \\
\text { Configuration }(\%)\end{array}$} \\
\hline & & & $X$ & $\mathrm{y}$ & $\mathrm{Z}$ & Total & \\
\hline 13-cis- $\beta$-carotene & 2.44 & 2.83 & 17.47 & -0.36 & -0.14 & 17.48 & $\mathrm{HOMO} \rightarrow \mathrm{LUMO}(94)$ \\
\hline 9-cis- $\beta$-carotene & 2.45 & 3.65 & 19.76 & 1.37 & -0.17 & 19.81 & $\mathrm{HOMO} \rightarrow \mathrm{LUMO}(94)$ \\
\hline phytofluene & 3.42 & 2.50 & 13.27 & 3.96 & 0.45 & 13.86 & $\mathrm{HOMO} \rightarrow \mathrm{LUMO}(98)$ \\
\hline trans- $\beta$-carotene & 2.43 & 3.92 & 20.62 & 0.87 & -0.09 & 20.63 & $\mathrm{HOMO} \rightarrow \mathrm{LUMO}(94)$ \\
\hline zeaxanthin & 2.37 & 4.10 & 21.15 & 2.69 & 0.13 & 21.33 & $\mathrm{HOMO} \rightarrow \mathrm{LUMO}(94)$ \\
\hline
\end{tabular}

